\title{
Gestational Sac Aspiration of Heterotopic Ectopic Pregnancy in a Cesarean Section Scar
}

\author{
Mohamed F. Mitwally, MD, ${ }^{1}$ Reda Alami, MD, ${ }^{2}$ Hafsa Albuarki, MBBS, ${ }^{3}$ Michael P. Diamond, MD, ${ }^{4,5}$ \\ Mostafa Abuzeid, MD, ${ }^{3,6,7}$ and Michael H. Fakih, $\mathrm{MD}^{3,7}$
}

\begin{abstract}
Background: This article describes a case of heterotopic pregnancy that included a normal twin intrauterine pregnancy and one cesarean section (CS) scar pregnancy diagnosed at 6 weeks of gestation. Ultrasound-guided aspiration of the ectopic gestational sac was performed, and the concurrent twin intrauterine pregnancy (IUP) was preserved successfully. The patient was a 50-year-old woman with secondary infertility. Case: The patient underwent in vitro fertilization and embryo transfer using a donor-egg program to achieve pregnancy with her current partner. At 6-weeks' gestation, she underwent a transvaginal ultrasound scan (US) examination showing a viable twin IUP with a third gestational sac with viable embryo located low within the anterior wall of the uterus. The appearance was consistent with a cesarean scar ectopic pregnancy. This was confirmed on a subsequent US 1 week later. She desired to continue the intrauterine pregnancy. US-guided aspiration of the cesarean scar ectopic pregnancy was attempted. The treatment was successful. Results: The twin pregnancy progressed without further complications. Conclusions: Heterotopic CS ectopic pregnancy can be successfully treated with transvaginal US-guided aspiration. (J GYNECOL SURG 29:317)
\end{abstract}

\section{Introduction}

$\mathbf{H}$ ETEROTROPIC PREGNANCY, the coexistence of an intraand extrauterine pregnancy, is an extremely rare event $(1$ in 30,000). However, the incidence of heterotopic pregnancy achieved by assisted reproduction has been reported to be as high as $1 \%-4 \% .1-3$ It is even more rare when the ectopic pregnancy is located in a previous cesarean section (CS) scar. The reported incidence is 1:18004 or 1:2216.5 Because of its rarity, there are no universal treatment guidelines to manage this condition, which carries a high risk of uterine rupture and uncontrolled hemorrhage. It usually presents a clinical dilemma when preservation of intrauterine pregnancy is desired. This article reports a case of heterotopic pregnancy that included a normal twin intrauterine pregnancy (IUP) and one ectopic pregnancy in a CS scar that was diagnosed at 6-week gestation. Ultrasound (US)-guided aspiration of the ectopic gestational sac was performed, and the concurrent twin IUP was preserved successfully

\section{Case}

A 50-year-old Caucasian female, gravida 5, para 2, with two early spontaneous miscarriages, one ruptured ectopic pregnancy, and a low transverse CS with a diagnosis of secondary infertility presented to the authors' unit in 2005 requesting to undergo a second cycle of in vitro fertilization and embryo transfer (IVF-ET) using a donor egg program with her current husband.

Four years earlier, she had undergone a successful IVF-ET using a donor egg program that resulted in a singleton term pregnancy delivered by a primary low transverse CS. Three

\footnotetext{
${ }^{1}$ Odessa Reproductive Medicine Center, Odessa, TX.

${ }^{2}$ Department of Obstetrics and Gynecology, Hurley Medical Center, Flint, MI.

${ }^{3}$ IVF Michigan, PC, Rochester Hills, MI.

${ }^{4}$ Division of Reproductive Endocrinology \& Infertility, Wayne State University School of Medicine, Detroit, MI.

${ }^{5}$ Detroit Regional Institute for Clinical and Translational Research, Detroit, MI.

${ }^{6}$ Division of Reproductive Endocrinology and Infertility, Department of Obstetrics and Gynecology, Hurley Medical Center, Flint, MI.

${ }^{7}$ Department of Obstetrics and Gynecology and Reproductive Biology, College of Human Medicine, Michigan State University, East Lansing, MI.

This abstract was presented at the 35th Annual Meeting of the American Association of Gynecological Laparoscopists (AAGL), Las Vegas, November 2006. Published as Mitwally MFM, Albuarki H, Elhammady E, Diamond MP, Abuzeid M, Fakih MH, Gestational sac aspiration of ectopic pregnancy in a cesarean section scar: Case report of successful management of heterotopic pregnancy following in-vitro fertilization [abstr]. J Minim Invasive Gynecol 2006;13(suppl):186.
} 


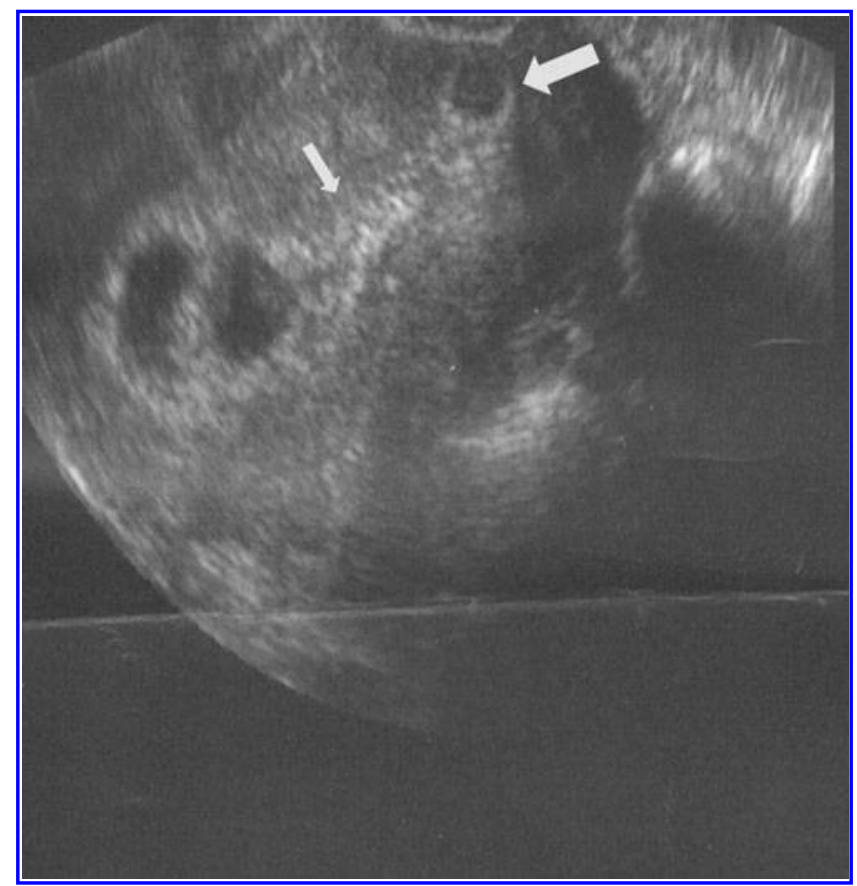

FIG. 1. Transvaginal ultrasound scan picture showing a viable twin intrauterine pregnancy (IUP) compatible with 6 weeks' gestation and a third ectopic gestational sac containing a viable embryo seen in the anterior wall of the lower uterine segment at the site of a previous cesarean section (CS) scar (large arrow). The distance between the edge of the gestational sac in the CS scar and the edge of the closer IUP gestational sac was approximately $35 \mathrm{~mm}$ (small arrow).

embryos were transferred into the uterine cavity under US guidance, and a pregnancy test was positive 2 weeks later. Transvaginal US examination at approximately 6 weeks' gestation revealed multiple gestation with a twin IUP and a third gestational sac implanted at the anterior wall of the lower uterine segment, at the site of the previous CS scar (Figs. 1 and 2). All three embryos had cardiac activity. The distance between the edge of the gestational sac in the CS scar and the edge of the closer IUP gestational sac was approximately $35 \mathrm{~mm}$ (Fig. 1). A diagnosis of heterotopic pregnancy in a CS scar was made. The patient had no discomfort, and her pelvic examination was unremarkable. The finding was relayed to the patient and her husband. After extensive counseling about the condition, its risks and treatment options, a conservative management strategy was adopted because of the patient's strong desire to preserve the twin IUP. Under intravenous sedation and sterile condition, a 16-gauge needle was used to aspirate the contents of the gestational sac in the CS scar under transvaginal US scan guidance. The patient tolerated the procedure well.

After 1 week, a repeat US showed a viable twin IUP and the persistence of a CS gestational sac, but no evidence of fetal pole or cardiac pulsations was seen at that time (Fig. 3). It was explained to the patient that trophoblastic tissue can be active in the absence of an embryo and, in turn, she was still at risk for possible complications. Transvaginal US was repeated 5 days later with the same findings reported in the previous examination, with no increase in size of the ectopic gestational sac and a normal viable twin IUP at 8-week

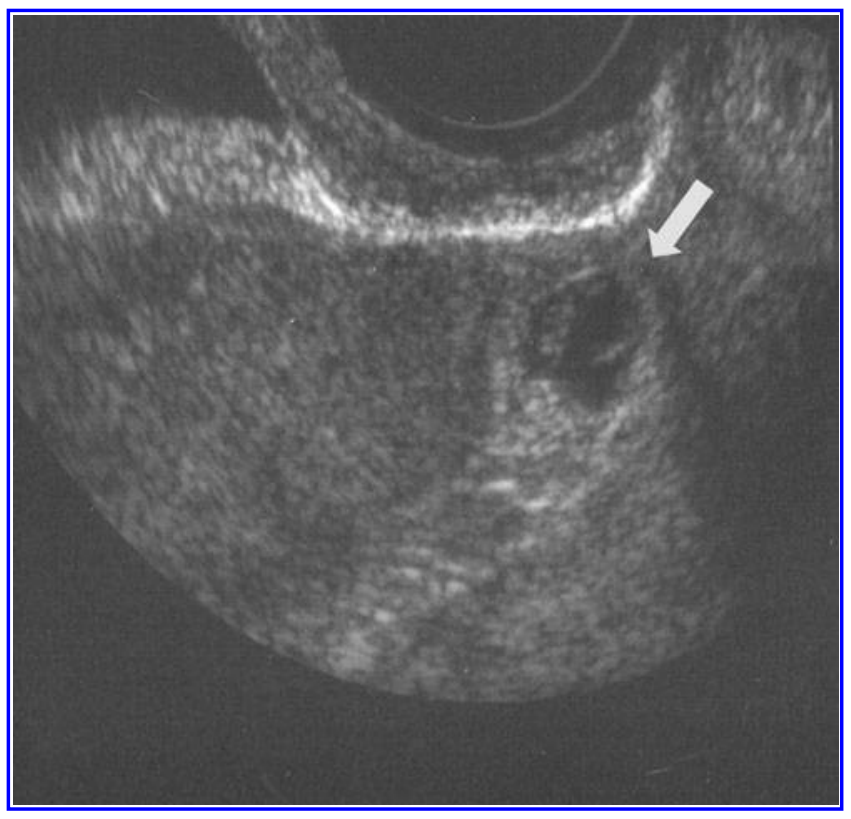

FIG. 2. Transvaginal ultrasound scan of an ectopic gestational sac containing a viable embryo located in the anterior wall of the lower uterine segment at the site of a previous cesarean section scar (arrow).

gestation. Four weeks later, another transvaginal US scan was performed, which revealed shrinkage and almost complete resorption of the gestational sac (Fig. 4). The twin IUP proceeded without further complications until 32-week gestation, when a repeat low transverse CS was performed because she had developed preeclampsia. At the time of her $\mathrm{CS}$, there was no evidence of dehiscence at the site of the cesarean scar. Following the delivery, the low transverse CS incision in the uterus was sutured using 0-Vicryl in two layers in continuous running manner.

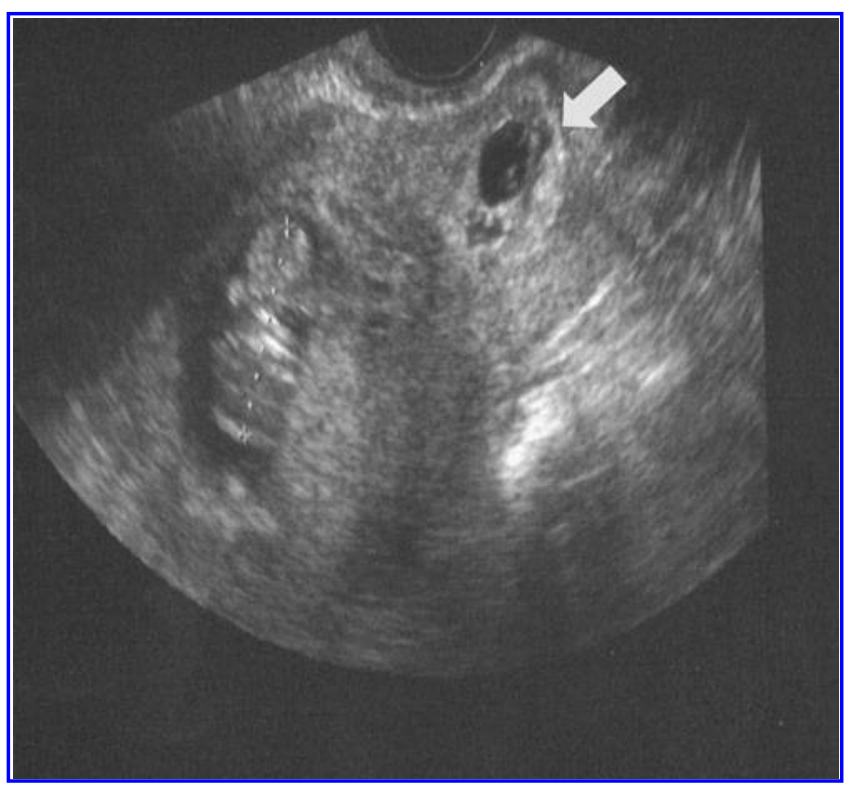

FIG. 3. Transvaginal ultrasound scan (US) scan of a repeat US scan showing a viable twin intrauterine pregnancy at 7 weeks' gestation and the persistence of cesarean section gestational sac, but no evidence of fetal pole (arrow). 


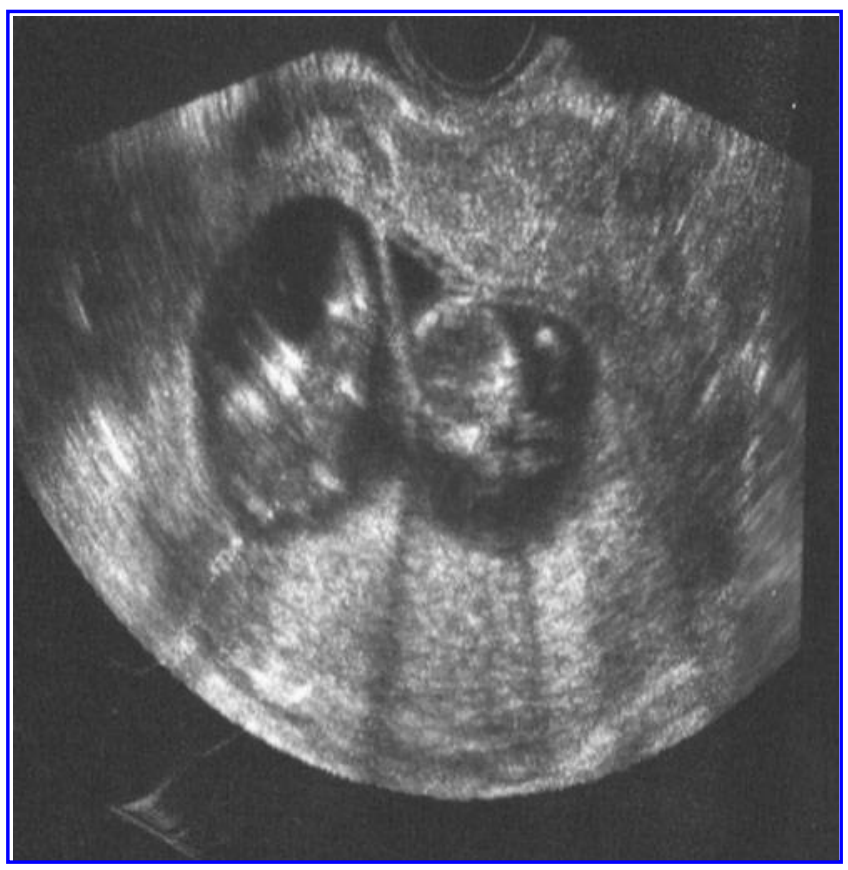

FIG. 4. Transvaginal ultrasound scan showing shrinkage and almost complete resorption of ectopic gestational sac in the cesarean section scar and a viable twin intrauterine pregnancy at 11 weeks' gestation.

\section{Discussion}

Ectopic pregnancy in a CS scar (CSP) is the implantation of a pregnancy within a cesarean fibrous tissue scar. It is the most rare form of ectopic pregnancies. The diagnosis and treatment have changed dramatically in the last 20 years. Before 1980, CSP would often present with life-threatening hemorrhage and uterine rupture requiring emergency hysterectomy. Now, it is commonly diagnosed on a first trimester US. The number of cases described in the literature appear to be much higher since 2002, presumably because of an increase in the number of cesarean procedures currently being performed, as well as the more widespread use of transvaginal US that enable an earlier detection of such pregnancies. The sonographic criterion for the diagnosis of CSP is the presence of gestational sac in the anterior part of the isthmic portion of the uterus. Occasionally CSP can be visualized in between the bladder and the gestational sac. Other diagnostic tools such as magnetic resonance imaging, cystoscopy, hysteroscopy, and laparoscopy can also be used.

Two possible conservative treatment options may be considered: medical or surgical. Both aim to eliminate the gestational sac and preserve the patient's fertility. Medical treatment with systemic or local injection of methotrexate (MTX) has been reported. Recently, Nawroth et al. also described the combined effect of local and systemic MTX administration with or without aspiration. ${ }^{6}$ Potassium chloride (KCL), or hyperosmolar glucose, or a hypertonic solution of sodium chloride has been used as well. ${ }^{3,7,8}$ Heterotopic pregnancy is the coexistence of IUP and extrauterine pregnancy. Its incidence has increased with the widespread use of assisted reproductive technology (ART). The diagnosis can be difficult; heterotopic pregnancies often escape early recognition because both serum $\beta$-human chorionic gonad- otropin and US can be misleading, and the diagnosis is not made until ectopic rupture with internal hemorrhage and shock. Gupta et al. reported a ruptured ectopic pregnancy in a CS scar at 9-week gestation necessitating laparotomy and hysterectomy. ${ }^{9}$ They concluded that early diagnosis is very important to avoid catastrophic hemorrhage and to avoid the need for surgery. ${ }^{9}$ The current authors believe that the successful outcome in the case described in this article can be attributed to early diagnosis and management.

Heterotopic CSP is extremely rare, and its occurrence in conjunction with a viable IUP and the patient's desire to keep the IUP make management very challenging. Salomon et al. described a heterotopic CSP with one embryo implanted into the uterine cavity, and the other located in the anterior isthmus wall that was successfully managed with KCL injection into the embryo to terminate the CSP at 8 weeks of gestation. ${ }^{10}$ Other reports have shown that during the injection of KCL, the embryo is sometimes pushed away from the needle and KCL diffuses into the amniotic sac. Consequently, it may diffuse to the adjacent sac and cause a toxic effect on the remaining embryo(s). A surgical option with laparotomy and excision of the cesarean ectopic from the lower uterine segment and repair was also proposed. ${ }^{11}$ The patient in the present report did not want to take any chance and emphasized the need for preserving the intrauterine twin pregnancy; therefore, the authors opted for the most conservative management possible.

To the authors' knowledge, the current case is the third reported heterotopic CSP, and the second to be successfully treated with US-guided aspiration. Hsieh et al. (2004) reported a similar case that was successfully treated with US-guided aspiration, and twin IUP was successfully preserved to be delivered at 32 weeks of gestation because of preterm labor. ${ }^{12}$

\section{Conclusions}

In view of the increasing number of CS being performed and the use of ART, obstetricians should be aware of the possibility of this type of heterotopic pregnancy. The present reinforces what was reported by Hsieh and suggests that a heterotopic CS ectopic pregnancy can be successfully treated with US-guided aspiration if diagnosis is made early in pregnancy.

\section{Acknowledgments}

The authors wish to thank Cheryl Anderson for preparation of the manuscript.

\section{Disclosure Statement}

The authors have no commercial, proprietary, or financial interest in the products or companies described in this article.

\section{References}

1. Dimitry ES, Subak-Sharpe R, Mills M, Margara R, Winston $R$. Nine cases of heteroscopic pregnancies in 4 years of invitro fertilization. Fertil Steril 1990;53:107.

2. Rizk B, Tan SL, Morcos S, Morcos S, et al. Heterotopic pregnancies following in-vitro fertilization and embryo transfer. Am J Obstet Gynecol 1991;164:161.

3. Marcus SF, Macnamee M, Brinsden P. Heteroscopic pregnancies after in vitro fertilization and embryo transfer. $\underline{\text { Hum }}$

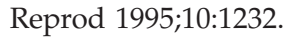


4. Jurkovic D, Hillaby K, Woelfer B, Lawrence A, Salim R, Elson CJ. First trimester diagnosis and management of pregnancies implanted into the lower uterine segment cesarean section scar. Ultrasound Obstet Gynecol 2003; 21:220.

5. Seow KM, Huang LW, Lin YH, Lin MY, Tsai YL, Hwang JL. Cesarean scar pregnancy: Issues in management. Ultrasound Obstet Gynecol 2004;23:247.

6. Nawroth F, Foth D, Wilhelm L, Schmidt T, Warm M, Romer $\mathrm{T}$. Conservative treatment of ectopic pregnancy in a cesarean section scar with methotrexate: A case report. Eur J Obstet Gynecol Reprod Biol 2001;99:135.

7. Clayton HB, Schieve LA, Peterson HB, Jamieson DJ, Reynolds MA, Wright VC. A comparison of heterotopic and intrauterine-only pregnancy outcomes after assisted reproductive technologies in the United States from 1999 to 2002. Fertil Steril 2007;87:303.

8. Verma U, Goharkhay N. Conservative management of cervical ectopic pregnancy. Fertil Steril 2009;91:671.

9. Gupta A, Daya S, Duhan N, Bishnoi S. Lower segment cesarean scar pregnancy: A cause of uterine rupture in the first trimester. J Gynecol Surg 2012;28:179.
10. Salomon LJ, Fernandez H, Chauveaud A, Doumerc S, Frydman R. Successful management of a heterotopic Caesarean scar pregnancy: Potassium chloride injection with preservation of the intrauterine gestation: Case report. Hum Reprod 2003;18:189.

11. Tolino A, Battista L, Chiacchio G, Rania E, Materazzo C, Palomba S. Cervico-isthmic pregnancy developing within the scar of a previous cesarean section: A case report. Gynecol Surg 2010;7:43.

12. Hsieh BC, Hwang JL, Pan HS, Huang SC, Chen CY, Chen $\mathrm{PH}$. Heterotopic Caesarean scar pregnancy combined with intrauterine pregnancy successfully treated with embryo aspiration for selective embryo reduction: Case report. Hum Reprod 2004;19:258.

Address correspondence to: Mostafa Abuzeid, MD Department of Obstetrics and Gynecology Hurley Medical Center

Two Hurley Plaza, Suite 209

Flint, MI 48503

E-mail: reprod1@hurleymc.com 